\title{
Reconstruction of large-scale peculiar velocity fields
}

\author{
Roya Mohayaee ${ }^{1}$, R. Brent Tully ${ }^{1,2}$ and Uriel Frisch ${ }^{1}$ \\ ${ }^{1}$ Observatoire de la Côte d'Azur, B.P.4229, F-06304 Nice Cedex 4, France \\ ${ }^{2}$ Institute for Astronomy, University of Hawaii, Honolulu, HI 96822, USA \\ Invited contribution to Colloquium, Cosmology: facts and Problems, \\ Collège de France, Paris 8-11, June 2004 \\ Eds. J.V. Narlikar \& J-.C. Pecker
}

December 29, 2018

\begin{abstract}
A reconstruction method for recovering the initial conditions of the Universe starting from the present galaxy distribution is presented which guarantees uniqueness of solutions. We show how our method can be used to obtain the peculiar velocities of a large number of galaxies, hence trace galaxies orbits back in time and obtain the entire past dynamical history of the Universe above scales where multi-streaming has not occurred. When tested against a $128^{3} \Lambda \mathrm{CDM}$ simulation in a box of $200 \mathrm{~h}^{-1} \mathrm{Mpc}$ length, we obtain $60 \%$ exact reconstruction on scales above $6 \mathrm{~h}^{-1} \mathrm{Mpc}$. We apply our method to a real galaxy redshift catalogue, the updated NBG (Nearby Galaxies), containing 1483 galaxies, groups and clusters in a radius of $30 \mathrm{Mpc} / \mathrm{h}$, and reconstruct the peculiar velocity fields in the local neighbourhood. Our reconstructed distances are wellmatched to the observed values outside the collapsed regions if $\Omega_{m}(t)=0.20 \exp (-0.26(t-13))$ where $t$ is the age of the Universe in Gyrs.
\end{abstract}

\section{Introduction}

Reconstruction of the initial condition of the Universe from the present distribution of the galaxies, brought to us by ever-more sophisticated redshift surveys, is an instance of the general class of inverse problems in physics. In cosmology this problem is frequently tackled in an empirical way by a forward approach. A statistical comparison between the outcome of an N-body simulation and the observational data is made, assuming that a suitable bias relation exists between the distribution of galaxies and that of dark matter. If the statistical test is satisfactory then the implication is that the initial condition assumed by the simulation is a viable one for our Universe, otherwise one changes the cosmological parameters until a statistical convergence between the observed and the simulated present Universe is achieved.

Since Newtonian gravity is time-reversible, one could integrate the equations of motions back in time and solve the reconstruction problem trivially, if in addition to their positions, the present velocities of the galaxies were also known. However, the peculiar velocities of only a few thousands of galaxies are known out of thousands whose redshifts have been measured. Thus, a second boundary condition, in addition to the present redshifts of the galaxies, has to be provided : as we go back in time the peculiar velocities of the galaxies vanish. Contrary to the forward approach 
where one solves an initial-value problem, in the reconstruction approach one is dealing with a twopoint boundary value problem (In this case, only the functional dependence of one of the boundary conditions is given, namely: time $\rightarrow 0$ then peculiar velocities $\rightarrow 0$ ). In the former, one has a unique solution but in the latter this is not always the case.

The question remains whether unique reconstruction can be achieved. In this work, we report on a new method of reconstruction (Frisch et al. 2002, Mohayaee et al. 2003, Brenier et al. 2003) which guarantees uniqueness.

\section{A brief review of previous approaches to reconstruction}

The history of reconstruction goes back to the work of Peebles who traced the orbits of the members of the Local Group back in time (Peebles 1989). In his approach, reconstruction was solved as a variational problem. Instead of solving Newton's equations of motion, one searches for the stationary points of the corresponding Euler-Lagrange action. In his first work (Peebles 1989) only the minimum of the action was considered. Later on, it was found that when the trajectories corresponding to the saddle-point of the action were taken, a better agreement between predicted and observed velocities could be obtained for the galaxies in the Local Group (peebles 1995). Thus, by adjusting the orbits until the predicted and observed velocities agreed, reasonable bounds on cosmological parameters were found (Peebles 1989) consistently favouring a low density Universe $\left(\Omega_{m}=0.1-0.2\right.$; noteworthy at a time when there was a common preference for $\left.\Omega_{m}=1\right)$.

Although rather successful (Shaya et al. 1995, Peebles et al. 2001) when applied to catalogues such as NBG (Tully 1988) and also to mock catalogues (Branchini, Eldar\& Nusser 2002), reconstruction with such an aim, namely establishing bounds on cosmological parameters using measured peculiar velocities, cannot be applied to larger galaxy redshift surveys which contain hundreds of thousands of galaxies for the majority of which the peculiar velocities are unknown. For large catalogues, the number of solutions become very large and not only uniqueness is completely lost but also one is never sure that the full solution space has been explored. In addition, numerical action-based codes are needed to solve the problem that challenges current computer capacities

A physical reason for multiple solutions is the collisionless nature of cold dark matter. Collisionless fluid elements can undergo multistreaming. Regions of multistream are bounded by caustics where the density is formally infinite and inside which the velocity field can have more than one value. This is a major obstacle to a unique reconstruction.

\section{Monge-Ampère-Kantorovich (MAK) reconstruction}

Reconstruction can be a well-posed problem for as long as we avoid multistream regions. The mathematical formulation of this problem is as follows (see Frisch et al. 2002, Mohayaee et al. 2003 and Brenier et al. 2003). Unlike most of the previous works on reconstruction where one studies the Euler-Lagrange action, we start from a constraint equation, namely the mass conservation,

$$
\rho(\mathbf{x}) d \mathbf{x}=\rho_{0}(\mathbf{q}) d \mathbf{q},
$$

where $\rho_{0}(\mathbf{q})$ is the density at the initial position, $\mathbf{q}$, and $\rho(\mathbf{x})$ is the density at the present position, $\mathbf{x}$, of the fluid element. The above mass conservation equation can be rearranged in the following form

$$
\operatorname{det}\left[\frac{\partial q_{i}}{\partial x_{j}}\right]=\frac{\rho(\mathbf{x})}{\rho_{0}(\mathbf{q})},
$$


where det stands for determinant and $\rho_{0}(\mathbf{q})$ is constant. The right-hand-side of the above expression is basically given by our boundary conditions: the final positions of the particles are known and the initial distribution is homogeneous, $\rho_{0}(\mathbf{q})=$ const. To solve the equation, we make the following hypotheses: the Lagrangian map $(\mathbf{q} \rightarrow \mathbf{x})$, is the gradient of a convex potential $\Phi$. That is

$$
\mathbf{x}(\mathbf{q}, t)=\nabla_{q} \Phi(\mathbf{q}, t) .
$$

The convexity guarantees that a single Lagrangian position corresponds to a single Eulerian position, i.e., there has been no multistreaming ${ }^{1}$. These assumptions imply that the inverse map $\mathbf{x} \rightarrow \mathbf{q}$ also has a potential representation

$$
\mathbf{q}=\nabla_{\mathbf{x}} \Theta(\mathbf{x}, t)
$$

where the potential $\Theta(\mathbf{x})$ is also a convex function and is related to $\Phi(\mathbf{x})$ by the Legendre-Fenchel transform (e.g. Arnold 1978)

$$
\Theta(\mathbf{x})=\max _{\mathbf{q}}[\mathbf{q} \cdot \mathbf{x}-\Phi(\mathbf{q})] \quad ; \quad \Phi(\mathbf{q})=\max _{\mathbf{x}}[\mathbf{x} \cdot \mathbf{q}-\Theta(\mathbf{x})]
$$

The inverse map is now substituted in (2) yielding

$$
\operatorname{det}\left[\frac{\partial^{2} \Theta(\mathbf{x}, t)}{\partial x_{i} \partial x_{j}}\right]=\frac{\rho(\mathbf{x})}{\rho_{0}(\mathbf{q})},
$$

which is the well-known Monge-Ampère equation (Monge 1781, Ampère 1820) The solution to this 222 years old problem has recently been discovered (Brenier 1987, Benamou and Brenier 2000) when it was realized that the map generated by the solution to the Monge-Ampère equation is the unique solution to an optimisation problem. This is the Monge-Kantorovich mass transportation problem (Kantorovich 1942), in which one seeks the map $\mathbf{x} \rightarrow \mathbf{q}$ which minimises the quadratic cost function

$$
I=\int_{\mathbf{q}} \rho_{0}(\mathbf{q})|\mathbf{x}-\mathbf{q}|^{2} d^{3} q=\int_{\mathbf{x}} \rho(\mathbf{x})|\mathbf{x}-\mathbf{q}|^{2} d^{3} x .
$$

A sketch of the proof is as follows. A small variation in the cost function yields

$$
\delta I=\int_{\mathbf{x}}[2 \rho(\mathbf{x})(\mathbf{x}-\mathbf{q}) \cdot \delta \mathbf{x}] d^{3} x,
$$

which must be supplemented by the condition

$$
\nabla_{\mathbf{x}} \cdot(\rho(\mathbf{x}) \delta \mathbf{x})=0
$$

which expresses the constraint that the Eulerian density remains unchanged. The vanishing of $\delta I$ should then hold for all $\mathbf{x}-\mathbf{q}$ which are orthogonal (in $L^{2}$ ) to functions of zero divergence. These are clearly gradients. Hence $\mathbf{x}-\mathbf{q}(\mathbf{x})$ and thus $\mathbf{q}(\mathbf{x})$ is a gradient of a function of $\mathbf{x}$.

Discretising the cost (7) into equal mass units yields

$$
I=\min _{j(\cdot)}\left(\sum_{i=1}^{N}\left(\mathbf{q}_{j(i)}-\mathbf{x}_{i}\right)^{2}\right) .
$$

\footnotetext{
${ }^{1}$ The gradient condition has been made in previous works (Bertschinger and Dekel 1989) on the reconstruction of the peculiar velocities of the galaxies using linear Lagrangian theory.
} 
The formulation presented in (10) is known as the assignment problem: given $N$ initial and $N$ final entries one has to find the permutation which minimizes the quadratic cost function. The cost function is indeed the minimum of an Euler-Lagrange action for inertial particles formulated in suitable space and time coordinates (Croft \& Gaztañaga 1997). If one were to solve the assignment problem (10) for $N$ particles directly, one would need to search among $N$ ! possible permutations, for the one which would have the minimum cost. However, advanced assignment algorithms exist which reduce the complexity of the problem from factorial to polynomial (e.g. see Hénon 1995 and Bertsekas 1998. Furthermore Hénon's adaptation of sparse and dense algorithm suitable for cosmological problems has a complexity of less than $N^{2.5}$ and has been used extensively in Mathis, Mohayaee \& Silk 2004.)

\section{Test against numerical simulation}

We have tested our reconstruction against numerical N-body simulation. We ran a $\Lambda$ CDM simulation of $128^{3}$ dark matter particles, using the adaptive $\mathrm{P}^{3} \mathrm{M}$ code HYDRA (Couchman et al. 1995). Our cosmological parameters are $\Omega_{m}=0.3, \Omega_{\Lambda}=0.7, h=0.65, \sigma_{8}=0.9$ and a box size of $200 \mathrm{Mpc} / \mathrm{h}$. The simulations started at high redshift, in this case at $z=70$. The results of our full box reconstruction are shown in Fig. 1. Once the assignment problem is solved the peculiar velocities can be simply evaluated using the zel'dovich approximation $\dot{\mathbf{x}}=f(\Omega) H(t) \times(\mathbf{x}-\mathbf{q})$ where $f(\Omega)=d \ln D / d \ln a$ is dimensionless linear growth rate, $D(t)$ is the amplitude of the growing mode today, $a$ is the cosmic scale factor and $H(t)$ is the value of the Hubble parameter (Zel'dovich 1970). The peculiar velocities can then be used to reconstruct the positions $\mathbf{x}$ of the particles at any desired redshift back in time : $\mathbf{x}(z)=\mathbf{q}+\left(D(z) / D_{0}\right)\left(\mathbf{x}_{0}-\mathbf{q}\right)$ where $\mathbf{x}_{0}$ is their present positions, given by the simulation and $D_{0}$ is the present value of $D$. The lower-inset of Fig. 1 shows the exact rate of reconstruction (when the separation between reconstructed and simulated positions of the particles is less than one mesh at $z=70$ ) to be more or less the same as that as the top left inset. The reason is that particles move very little from the grid positions at high redshifts. However, a comparison between the two histograms demonstrates that yet another Zel'dovich approximation which is involved in getting from grid positions to positions at $z=70$ does not decrease the success of our reconstruction. (For detailed tests against simulations and reconstruction of statistics of the primordial density field, e.g. works on issues such as non-Gaussianity, see Mathis, Mohayaee \& Silk 2004). For scales below 2 Mpc corresponding to smallest scale probed by reconstruction whose results are given in Fig. 1, the exact reconstruction rate is about $18 \%$ due to severe multistreaming at these scales. On larger scales of about $5 \%$ this rate increases to about $60 \%$.

Outside collapsed regions the reconstructed peculiar velocities match well those simulated as shown in Fig. 2. The primordial density field evaluated using these velocities also matches extremely well the simulated one as demonstrated in the lower panel of Fig. 2 (we thank S. Colombi for providing us with the lower panel of Fig. 2).

\section{$5 \quad$ Application to real galaxy catalogues}

We have applied our MAK method to the updated NBG catalogue (Tully 1988), now including 3300 galaxies within $3,000 \mathrm{~km} \mathrm{~s}^{-1}$. Other more extensive catalogues are available but this catalogue provides good completion within the specified volume, which is sufficient in depth for present purposes. The NBG has the important value added feature of the detailed assignment of all objects to homogeneously identified groups and filamentary structures. The zone of Milky Way 


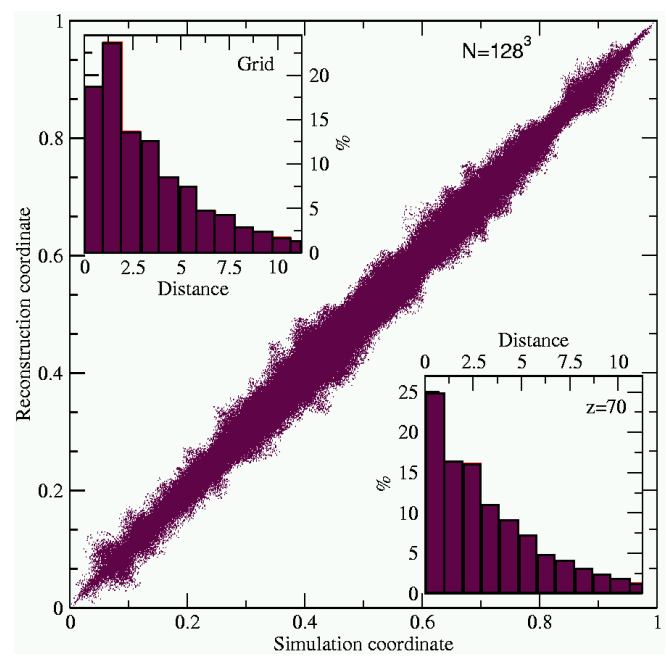

Figure 1: In the scatter plot, the dots near the diagonal are a scatter plot of reconstructed initial points versus simulation initial points for a grid of size $1.5 \mathrm{Mpc} / \mathrm{h}$ with more than 2 million points. The scatter diagram uses a quasi-periodic projection coordinate $\tilde{\mathbf{q}} \equiv\left(q_{x}+\sqrt{2} q_{y}+\sqrt{3} q_{z}\right) /(1+\sqrt{2}+\sqrt{3})$ which guarantees a one-to-one correspondence between $\tilde{\mathbf{q}}$ values and points on the regular Lagrangian grid. The upper left inset is a histogram (by percentage) of distances in reconstruction mesh units between such points; the first bin corresponds to perfect reconstruction; the lower-inset is a similar histogram for reconstructed points at $z=70$. The points at $z=70$ are obtained by using Zel'dovich approximation to push particles back in time once their grid position has been reconstructed. Perfect reconstruction of about $18 \%$ is achieved in both histograms on scales of about $2 \mathrm{Mpc}$. On mesh sizes of about $6 \mathrm{Mpc} / \mathrm{h}$ this rate increases to about $60 \%$.

avoidance is shrinking as new surveys are integrated but before a dynamical model can be computed something must be done to account for galaxies lost due to obscuration. In this work, fake galaxies were created by reflection of objects at nearby higher latitudes in sufficient numbers to achieve the average density for the volume. Another correction to the catalogue is one that accounts for incompleteness with distance. The correlation with mass is with the quantity of blue light. Light is lost from the catalogue as galaxies become increasingly excluded with distance. Fortunately the problem is not extreme over the limited range of this study. Selection function corrections to luminosity range from unity at less than $10 \mathrm{Mpc}$ (inside which there is completion because a low luminosity clip is imposed at $M_{B}=-16$ ) to only a factor 2.4 at $3000 \mathrm{~km} / \mathrm{s}$. The second observational component is a catalogue of galaxy distances. In all, there are over 1400 galaxies with distance measures within the $3,000 \mathrm{~km} \mathrm{~s}^{-1}$ volume. In the present study, distances are averaged over groups because orbits cannot meaningfully be recovered on sub-group scales. The present NBG catalogue is assembled into 1234 groups (including groups of one) of which 633 have measured distances.

This catalogue of galaxy positions, luminosities, and distances provides the basis for orbit reconstructions using MAK procedures. The distances, $d$, permit an extraction of peculiar velocities $V_{p e c}=V_{g s r}-d \mathrm{H}_{0}$ where $V_{g s r}$ is the observed velocity of an object in the galactic standard of rest.

For MAK reconstruction the particles must all have the same mass since all the particles on the initial grid must be equal and each orbit reconstruction has equal weight. Consequently the endpoint elements must be broken up by different amounts depending on their supposed relative masses. In the first approximation of constant mass-to-light ratio $M / L$ then the elements are simply broken into a number of particles that depends on $L_{i}$. The unit size is chosen to correspond 

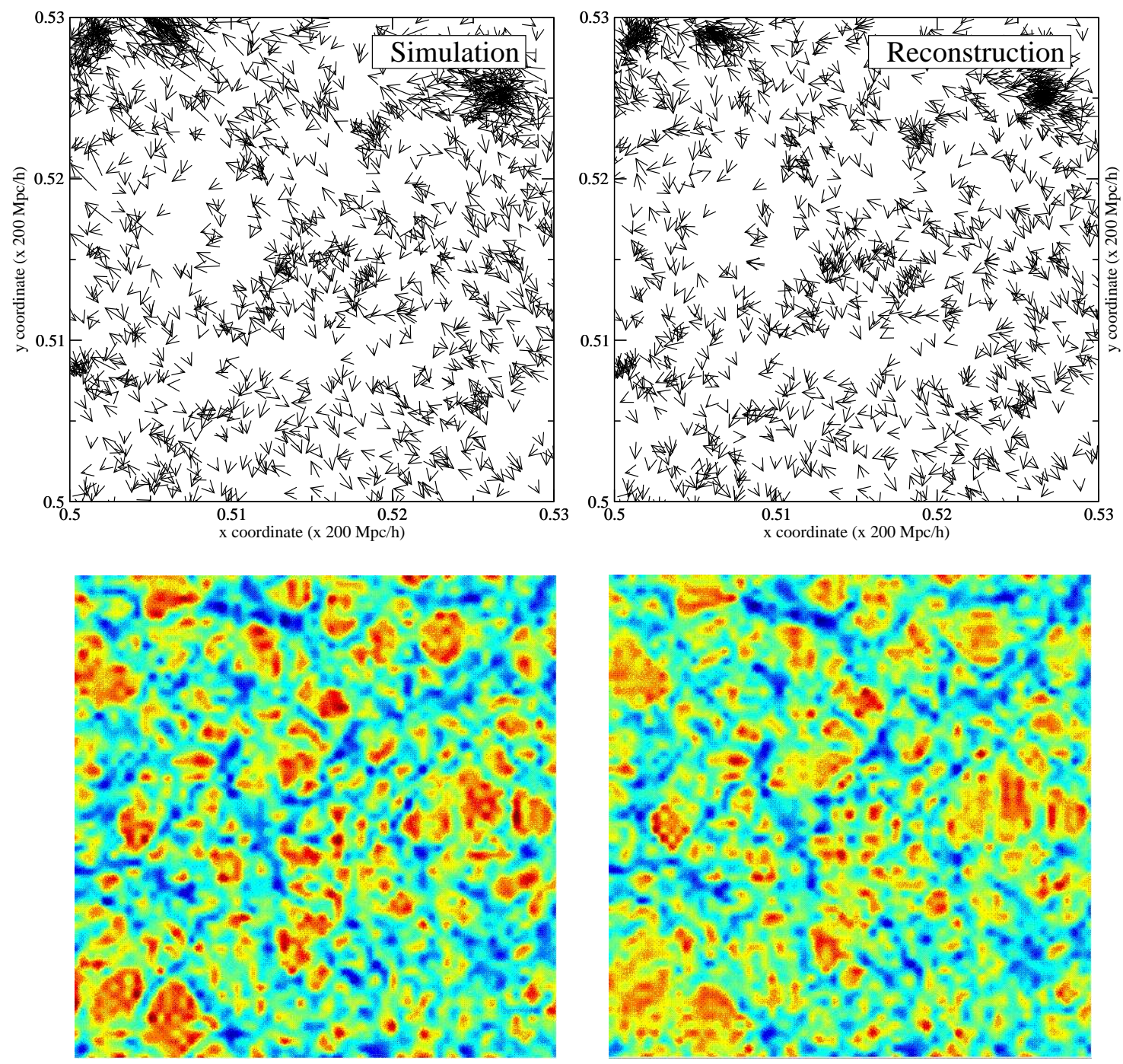

Figure 2: Top panel: simulated (left panel) and the reconstructed (right panel) velocity field are shown for a thin $6 \mathrm{Mpc} / \mathrm{h}$ slice in $x$ and $y$ direction and full-box projection in the $z$ direction of the simulation box. The reconstruction works extremely well outside dense/collapsed regions. bottom panel: Simulated density field (left panel) and the reconstructed (right panel) are shown for a thin slice cut of the simulation box. Red colour corresponds to denser regions and dark blue (lighter) are the void regions (cloud-in-cell interpolation is used). (Upper and lower panels do not correspond to the same slices of the simulation box.) 

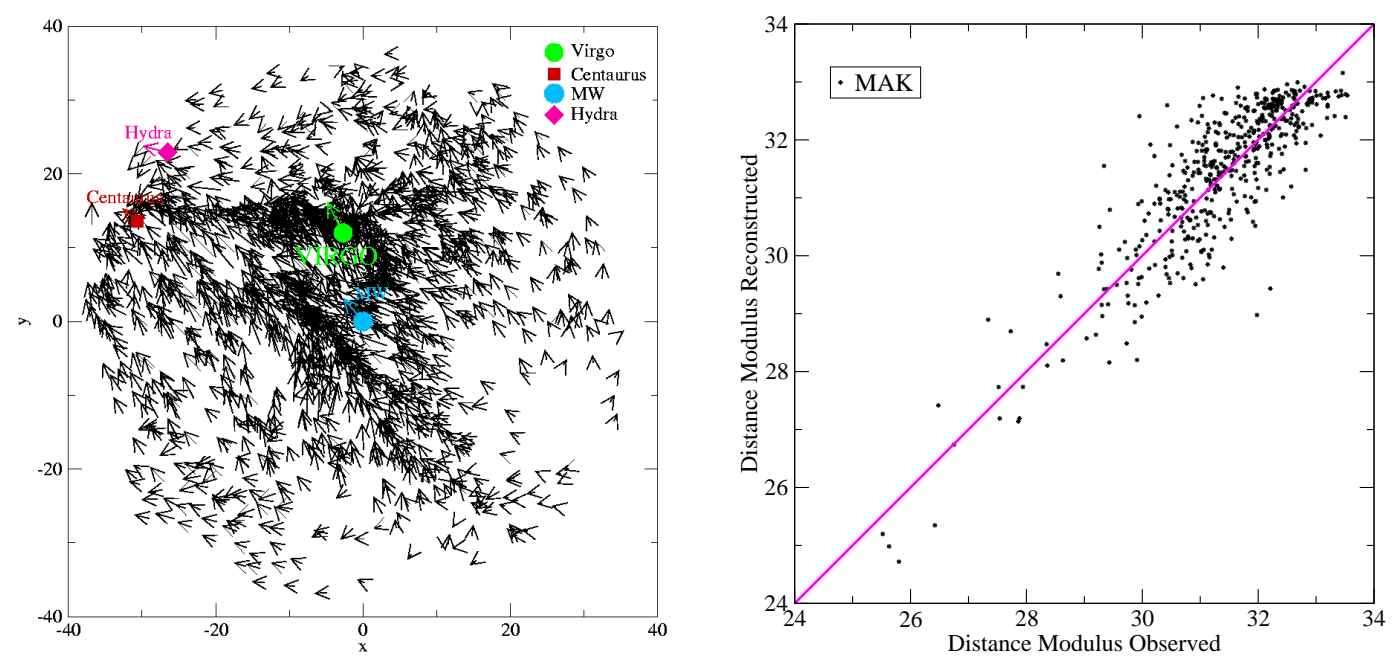

Figure 3: Left plot: Velocity field of objects in NBG catalogue obtained by MAK reconstruction is shown. Large-scale flow towards the great attractor is visible, which overshadows infall into the Virgo cluster. The supergalactic coordinates $x$ and $y$ are used. Right panel: is the scatter plot between MAK reconstructed distance modulus and that given by observations for the 663 objects with measured distances in this catalogue.

to $10^{9} L_{\odot}$, the faint end cutoff of the catalogue. The elements are all located in redshift space (i.e., at their positions on the sky and at a distance inferred from their velocities). However the breakup into particles for the MAK reconstruction does not preserve the velocity distortion from real positions within elements; $i$. e., on sub-group scales. As we have demonstrated in the previous section, the MAK reconstructions of N-body simulations demonstrates good recovery of orbits on scales greater than $5 h^{-1} \mathrm{Mpc}$ but clearly orbits cannot be recovered in shell-crossing regions.

The orbit of an element is defined by the center of mass of all the constituent particles as a function of time. The relationship between redshift and real space is estimated using the Zel'dovich approximation $\mathbf{v}=f(\Omega)(\mathbf{x}-\mathbf{q})$ where $\mathbf{v}$ is the peculiar velocity vector, $\mathbf{x}$ is the current Eulerian position, $\mathbf{q}$ is the initial Lagrangian position, and $f(\Omega) \sim 1+b / \Omega_{m, 0}^{4 / 7}+\left(1+\Omega_{m, 0} / 2\right) \Omega_{\Lambda, 0} / 70$ and $b$ is the bias factor which we take equal to 1 . In principle with our methodology, a variable bias can be obtained by varying $M / L$ with location. In this discussion, the same $M / L$ is assigned to all objects.

Once the particles are reconstituted into the catalogue elements, a specific model defines positions that can be tested against observed positions. In Figures 3, we show two MAK results. The left panel is the peculiar velocity field reconstructed by MAK of all the entries in the NBG catalog. There is a clear flow towards the great attractor as expected. The right panel shows a scatter plot of reconstructed versus observed distance moduli, $\mu_{i}=5 \log d_{i}+25$. The scatter is mainly due to poor reconstructions near big clusters such as Virgo. In the infall region of Virgo, one is in the highly non-linear regime and moreover in a triple-valued region due to redshift space distortion. In this region, velocities deviate significantly from Hubble flow and MAK reconstruction does not necessarily find the right solution. (For reconstruction in the infall region, used for determination of mass of Virgo cluster, see Tully \& Mohayaee 2004.) 


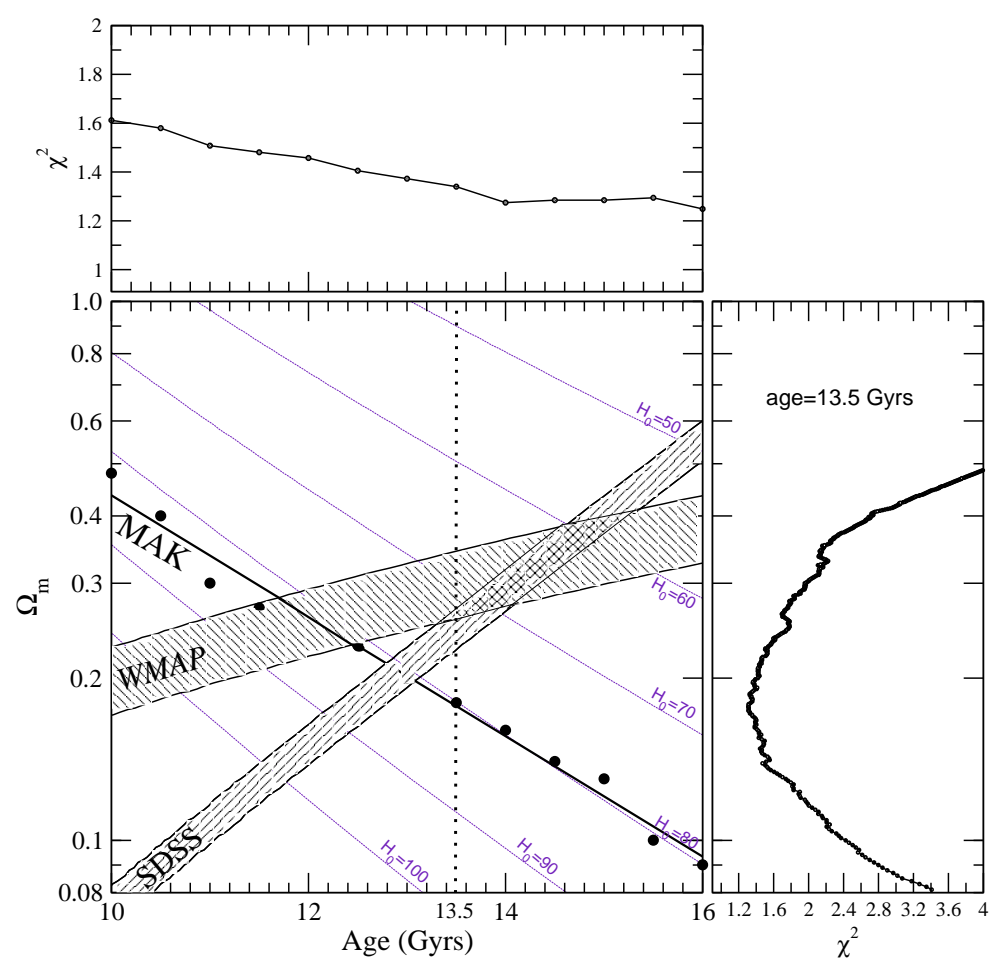

Figure 4: Constraints on the parameters $\Omega_{m}$ and age of the Universe given by MAK, WMAP, and SDSS. The $2 \sigma$ constraints from WMAP and SDSS are given as shaded bands. The minimum of the $\chi^{2}$ trough with the MAK reconstruction is given as the heavy solid line. Hubble constant contours are superposed as light lines. The two side panels illustrate aspects of $\chi^{2}$ with the MAK reconstruction. In the top panel, the minimum value of $\chi^{2}$ is shown at each age (ie, at the location of the heavy solid line). In the right side panel, the values of $\chi^{2}$ are shown for the range of $\Omega_{m}$ considered for the specific age $t=13.5 \mathrm{Gyr}$ (ie, the trace indicated by the vertical dotted line). It is seen that there is reasonable agreement between the three methodologies in the vicinity of $t=12-14$ Gyrs, $h=0.8$ and $\Omega_{m}=0.2-0.3$.

The overall MAK reconstruction can be evaluated by a $\chi^{2}$ estimator. We evaluate the median value for the $\chi_{i}^{2}$; between measured and observed distance moduli

$$
\chi_{i}^{2}=\left(\mu_{\text {observed }}-\mu_{M A K}\right)^{2} / \epsilon_{i}
$$

where $\epsilon_{i}$ is the error assigned to $\mu_{\text {observed }}$ which is the observed distance modulus of galaxy (or group or cluster) $i$ in the catalogue. Values of $\chi_{i}^{2}$ can be determined for the 633 objects in the catalogue with distance measures for a given choice of density parameter $\Omega_{m}$ and age $t$.

In this study we have only considered flat topologies. We assume that $\Omega_{\Lambda}=1-\Omega_{m}$ where $\Omega_{\Lambda}$ is a measure of the energy density of the Universe. With this constraint, there is a fixed relation between $\Omega_{m}, \mathrm{H}_{0}$, and the age of the Universe, $t$, such that if two of these parameters are specified then the third is defined: $h=(1 / \mathrm{t})(2 / 3)\left(1 / \sqrt{\left(1-\Omega_{m}\right)}\right) \log \left(\left(1+\sqrt{\left(1-\Omega_{m}\right)}\right) / \sqrt{\left(\Omega_{m}\right)}\right) 9.78$ where $h=H / 100$.

Constraints on the parameter space $\left(\Omega_{m}, t\right)$ are summarized in Fig. 4. The two broad bands locate the $95 \%$ confidence limits provided by WMAP spatial fluctuation and SDSS power spectrum 
studies (Spergel et al. 2003; Tegmark et al. 2004). The heavy solid line indicates the locus of $\chi^{2}$ minima as a function of age from the MAK reconstructions. This line is described by the equation $\Omega_{m}(t)=0.20 \exp (-0.26(t-13))$ with age $t$ in Gyr. The right panel illustrates the dependence of $\chi^{2}$ values on $\Omega_{m}$ at the fixed age of $t=13.5$ Gyr. The top panel shows the weak dependence of $\chi^{2}$ on age at the $\chi^{2}$-minimum trough defined by the heavy solid line. The overall minimum along this trough is reached at 17 Gyr. Overall with Fig. 4 two important points are to be noted. First, the uncertainties resulting from the MAK analysis are almost orthogonal to the WMAP and (especially) the SDSS constraints. Second, the three results intersect, resulting in concordance with the cosmological parameters $t=13.2 \pm 0.8 \mathrm{Gyr}, \Omega_{m}=0.25 \pm 0.05$, and $\mathrm{H}_{0}=77 \pm 5$.

In conclusion, we have demonstrated that our MAK reconstruction scheme guarantees uniqueness on large scales and can be applied to large datasets containing millions of objects. It is now being used with real data for the reconstruction of large-scale velocity fields. The method has been tested against numerical simulations and been shown to recover the peculiar velocities of a large number of galaxies with a high success rate (taking the simulation dark matter particles to trace galaxies). We have also shown that MAK can be applied to real data and reconstructed peculiar velocity fields in the Local Supercluster. The best reconstruction fits obey the relationship $\Omega_{m}(t)=0.20 \exp (-0.26(t-13))$, where $t$ is the age of the Universe in Gyrs. This fit intersects the WMAP and SDSS results within their $2 \sigma$ uncertainties in the range $t: 13-13.5$ Gyrs, whence $\Omega_{m}=0.2-0.3$.

Materials presented in Section 4, are parts of an ongoing collaboration with M. Hénon, S. Colombi and H. Mathis. Materials presented in Section 5 are parts of collaborations with J. Peebles, S. Phleps and E. Shaya. We also thank J. Colin, S. Matarrese and A. Sobolevskii for discussions and comments. R.M. is supported by a European Marie Curie fellowship HPMF-CT2002-01532. B.T. is partially supported by the BQR program of the Observatoire de la Côte d'Azur.

\section{References}

[1] Ampère A.-M. 1820, Mémoire concernant ... l'intégration des équations aux différentielles partielles du premier et du second ordre, Journal de L'École Royale Polytechnique 11, 1

[2] Arnold V.I. 1978, Mathematical Methods of Classical Mechanics (Springer, Berlin)

[3] Benamou J.-D. \& Brenier Y. 2000, The optimal time-continuous mass transport problem and its augmented Lagrangian numerical resolution, Numer. Math. 84, 375 (www.inria.fr/rrrt/rr-3356.html)

[4] Bertschinger E. \& Dekel A. 1989, Recovering the full velocity and density fields from large-scale redshift-distance samples, Astrophys. J. 336, L5

[5] Bertsekas D.P. 1998, Network Optimisation: Continuous and Discrete Models (Athena Scientific) \{Auction algorithm also available at http://web.mit.edu/dimitrib/www/auction.txt\}

[6] Branchini E., Eldar A. \& Nusser A. 2002, Peculiar velocity reconstruction with fast action method: tests on mock redshift surveys, Mon. Not. R. Astron. Soc. 335, 53

[7] Brenier Y. 1987, Décomposition polaire et réarrangement monotone des champs de vecteurs, $C$. R. Acad. Sci. Paris 305, 805 
[8] Couchman H.M.P., Thomas P.A. \& Pearce F.R. 1995, Hydra: an Adaptive-Mesh Implementation of $\mathrm{P}^{3} \mathrm{M}-\mathrm{SPH}$, Astrophys. J. 452, 797

[9] Croft R.A. \& Gaztañaga E. 1997, Reconstruction of cosmological density and velocity fields in the Lagrangian Zel'dovich approximation, Mon. Not. R. Astron. Soc. 285, 793

[10] Frisch U., Matarrese S., Mohayaee R. and Sobolevskii A. 2002, A reconstruction of the initial conditions of the Universe by optimal mass transportation, Nature 417, 260

Mohayaee R., Frisch U., Matarrese S. \& Sobolevskii A. 2003, Reconstruction of the primordial Universe by a Monge-Ampere-Kantorovich optimisation scheme, Astron. E3 Astrophys. 406, 393

Brenier Y., Frisch, U., Hénon M., Loeper, G. Matarrese, S. Mohayaee, R. \& Sobolevskii A. 2003, Reconstruction of the early Universe as a convex optimization problem, Mon. Not. R. Astron. Soc. 346, 501

[11] Hénon M. 1995, A mechanical model for the transportation problem, in Compte Rendu de l'Academie des Sciences 321, 741, A detailed version including an optimization algorithm is available at http://arXive.org/abs/math.OC/0209047

[12] Kantorovich L. 1942, On the translocation of masses, C.R. (Doklady) Acad. Sci. URSS (N.S.) 37, 199

[13] Mathis H., Mohayaee R. \& Silk J. 2004, Reconstructions of primordial CDM density fields, to be submitted to Mon. Not. R. Astron. Soc.

[14] Monge G. 1781, Mémoire sur la théorie des déblais et remblais, Hist. Acad. R. Sci. Paris, 666

[15] Peebles P.J.E. 1989, Tracing galaxy orbits back in time, Astrophys. J. 344, L53

[16] Peebles P.J.E. 1995, Mass of the Milky Way and Redshifts of the Nearby Galaxies, Astrophys. J. 449, 52

[17] Shaya E.J., Peebles P.J.E. and Tully R.B. 1995, Action Principle Solutions For Galaxy Motions Within $3000 \mathrm{Km} / \mathrm{s}$, Astrophys. J. 454, 15

Peebles P.J.E, Phelps S.D., Shaya E.J. \& R.B. Tully 2001 , Radial and Transverse Velocities of Nearby Galaxies, Astrophys. J. 554, 104

[18] Spergel D.N. et al. 2003, First-Year Wilkinson Microwave Anisotropy Probe (WMAP) Observations: Implications For Inflation, Astrophys. J. S. 148, 175

[19] Tegmark et al. 2004, Cosmological parameters from SDSS and WMAP, Astrophys. J. 607, 655

[20] Tully R. R.B. 1988, Nearby Galaxies Catalog, Cambridge University Press (Cambridge, UK)

[21] Tully R.B. \& Mohayaee Roya 2004, Action Model of Infall into the Virgo Cluster, astroph/0404006, in "Outskirt of Galaxy Clusters", the proceedings of IAU Colloquim No. 195,2004

Mohayaee Roya \& Tully R.B. 2004, The mass of the virgo cluster and surrounding supercluster, work in progress

[22] Zel'dovich Ya.B. 1970, Gravitational instability: an approximate theory for large density perturbations, Astron. 85 Astrophys. 5, 84 